\title{
Elasto-thermoelectric non-linear, fully coupled, and dynamic finite element analysis of pulsed thermoelectrics
}

\author{
J.L. Pérez-Aparicio*a, R. Palma ${ }^{b}$, P. Moreno-Navarro ${ }^{a}$ \\ ${ }^{a}$ Department of Continuum Mechanics $\mathcal{E}$ Theory of Structures, Universitat Politècnica de València, Spain \\ ${ }^{b}$ Department of Mechanical Engineering $\mathcal{G}$ Construction, Universitat Jaume I, Castellón de la Plana, Spain
}

\begin{abstract}
This paper presents a numerical study on the influence of pulsed electric signals applied to the overcooling of thermoelectric devices. To this end, an experimental setup taken from the literature and a commercial cell are simulated using a complete, specially developed research finite element code. The electro-thermal coupling is extended to include the elastic field, demonstrating that the increment of cooling can produce mechanical failure. Numerical results are developed and the variation of overcooling versus pulse gain and versus duration is validated towards a new analytical expression and the experimental data. The issue of optimal intensity at steady-state is also newly developed. Thermal and mechanical trends are presented using constant and variable (with temperature) material properties for a single thermoelement. While some of the first trends are similar to those of published works, others are different or directly new, all closer to those of the experiments. The mechanical results have not been thoroughly studied until recently. The three-dimensional finite element mesh includes non-thermoelectric materials that are fundamental for the current study. Distribution of stresses during steady and transient states are shown inside the thermoelement, for five components and for the combined Tresca stress. Concentrations at corners of the lower side appear close to the cold face. Due to these concentrations, 27-node isoparametric, quadratic brick elements are used. It is shown that the mechanical field is an important factor in the design of pulsed thermoelectrics, since for practical applications the stress levels are close or slightly above the admissible limits.
\end{abstract}

Keywords: Pulsed thermoelectric materials, Joule, Peltier, Thomson, Thermal stresses, Dynamic analytical solution, Optimal intensity, Stress distributions

\section{Introduction}

Thermoelectric (TE) devices under electric pulses are denominated Pulsed Thermoelectrics (PT's, for short PTs). PTs enhance the cooling power due to the increase of electric intensity: the Joule heat takes a longer time to reach the cold face than the Peltier cooling, thus reducing the minimum temperature $T$ with respect to that obtained in the steady-state situation. The different influence of both effects is due to the nature of Peltier, that is a convective effect concentrated at interfaces, while Joule is a source bulk effect distributed throughout the TE volume.

PTs are currently applied in electronic devices that need to be over-refrigerated for short intervals of time.

* Corresponding author. Tel: +34 96 3877000; fax: +34 96 3877189. E-mail address: jopeap@mes.upv.es
For example, they are mounted in laser gas sensors used in compliance monitoring and process control; see [1] for a complete review on thermoelectric applications.

To the best of our knowledge, the first study on PTs was developed by [2]. At present there are many experimental, analytical and numerical works in the literature, in particular experimental investigations were presented in [3] and [4]. Analytically, the pulse shape influences on the cooling power were studied in [5] considering constant (with respect to temperature, $\mathrm{CP}$ ) material properties. In addition, a temperature-entropy diagram based on thermodynamic considerations was developed in [6]. Numerically, the influence of the pulse shape and of the TE length on the PTs outputs was studied in [7] using a commercial Finite Element (FE) software, and in [8] using the Finite Difference method. In the last two works CP were again used, therefore the Thomson effect was not included (see Section 5.2).

In respect of analytical solutions, there are published 
works such as the static unidimensional model developed in [32] with CP and VP hypothesis. It includes the Thomson effect and some basic graded material calculation. Also, the dynamic unidimensional model with CP solved using the Sturm-Liouville eigensystem method of [33]; the accuracy of the resolution is based on the number of considered eigenvalues and can be computationally expensive. The current analytical solution is also unidimensional and dynamic with $\mathrm{CP}$ but the Laplace anti-transfromation is much faster.

In spite of the large number of works on PTs, we believe that two aspects remain unsolved: i) no complete studies on the mechanical behavior are available, even if the strong temperature gradients cause thermal stress concentrations, ii) no mathematical optimizations of the pulse shape and of the TE geometry have been done. The lack of published papers on PTs optimization is in contrast to the relatively large number on optimization of classical TE devices, see [9], [10] and [11].

Some studies on "three-field" (thermal, electric and mechanical) couplings using the FE have been published, but for steady-state situations. In [12], [13] calculations were performed with the commercial software COMSOL; a maximum stress of $37 \mathrm{MPa}$ was obtained using the Von Mises failure criterion although under a very high cold face temperature $T_{c}$. In [14], [15], [16], the also commercial ANSYS calculated in two stages due to the absence of complete coupling: a thermoelectric problem was solved first and then the temperature distributions were applied to a thermoelastic problem. The first reference concluded that higher weld thicknesses improve the mechanical efficiency of TEs, but in the current work it will be shown that this increase penalizes the overcooling and obviously increments the electric resistivity. In the second article, it was calculated that the studied cell could mechanically withstand a temperature difference of $100{ }^{\circ} \mathrm{C}$ although under a high $T_{c}$. The third reference indicates that significant stresses appeared in the TE corners, that again can be relaxed with thick welds to sustain plastic deformations. In general, the commercial software methodology is very computationally expensive and it would be difficult to apply it to the problem of PTs under dynamics and optimization, specially if a fine FE mesh is used to capture stress concentrations.

Both commented shortcomings could be due to the lack of fully nonlinear, coupled and dynamic numerical formulations implemented in available computer codes. To fill in part this gap, the authors have developed several complete nonlinear and dynamic formulations for thermoelectricity, [17], [18], [19], elastothermoelectricity [20] and fully coupled "four-field" (including magnetic) in [21]. Also, they are working on a one-dimensional (1D) formulation with beam elements for TEs to study their mechanical response minimizing computation time, [22].

While the optimization is the purpose of other ongoing works, [23], [24], the main aim of this paper is related to study the complete response of TE taking into account the electric, thermal and mechanical fields for which several new contributions are presented. First, in Section 2 the geometry, material and dynamics of the problem are established. In Section 3, a summary of the coupled and dynamic formulation, both thermodynamic and FE is presented. The formulation follows the steps developed in [21] and includes effects that are not always present in the study of thermoelectric devices, such as Thomson, Biot and the coupling with the mechanical field. The section also incorporates relevant aspects on the FE mesh, computer running and the implementation in the research code FEAP, [25]. The calculation of the optimal electric intensity to feed the thermocouple (TC) during steady-state is presented in Section 4, not only with $\mathrm{CP}$ but also with variable material properties (VP) function of the temperature field. In Section 5 the FE algorithm is validated with experimental and semi-analytical methods. The first is towards empirical relations taken from experiments by [4], and the second towards a simplified analytical solution with $\mathrm{CP}$ based in the Laplace transform. Finally, in Section 6 a thorough study of thermal stresses present in the TC is discussed. Due to the higher difficulty of the mechanical field numerical analysis (with respect to those of thermal and electrical), special care is taken for the study of stresses and strains. Also given the three dimensionality of the problem, a complete discussion of the five relevant stress components and the Tresca equivalent one is included.

\section{Data and variables of the problem}

The geometry of a typical TC has been thoroughly described in the literature; in this work we mainly analyze a Peltier cell similar to CP1.4-127-045 cell (but with different length $l_{3}$ ), Fig. 1, currently manufactured by [26] and described in [20]. The geometrical model includes, besides the p- $B i_{2} T e_{3}$, copper $C u$, alumina $\mathrm{Al}_{2} \mathrm{O}_{3}$ and tin-lead solder $\mathrm{SnPb}$ materials. The last three are not very relevant for the electric field but important to properly simulate the thermal inertias (see discussion in Fig. 9) and fundamental for the calculation of stresses as will be shown in Section 6.

Taking advantage of repetitions, only the left half of the TC comprising the p-type TE is considered 


\begin{tabular}{|c|c|c|c|c|c|}
\hline Property & Unit & $\mathrm{Al}_{2} \mathrm{O}_{3}$ & $\mathrm{Cu}$ & $\mathrm{SnPb}^{3}$ & $\mathrm{Bi}_{2} \mathrm{Te}_{3}$ \\
\hline \hline$\rho \times 10^{3}$ & $\mathrm{~kg} / \mathrm{m}^{3}$ & 3.57 & 8.96 & 7.31 & 7.53 \\
\hline$c_{p} \times 10^{2}$ & $\mathrm{~J} / \mathrm{kg} \mathrm{K}$ & 8.37 & 3.85 & 2.26 & 5.44 \\
\hline$\alpha_{T} \times 10^{-6}$ & $1 /{ }^{\circ} \mathrm{C}$ & 5.00 & 17 & 27 & 16.8 \\
\hline$\lambda \times 10^{10}$ & $\mathrm{~N} / \mathrm{m}^{2}$ & 16.34 & 7.16 & 3.25 & 6.71 \\
\hline$\mu \times 10^{10}$ & $\mathrm{~N} / \mathrm{m}^{2}$ & 15.08 & 4.39 & 1.68 & 1.68 \\
\hline$\kappa$ & $\mathrm{W} / \mathrm{K} \mathrm{m}$ & 35.3 & 386 & 48 & $\kappa(T)$ \\
\hline$\gamma \times 10^{6}$ & $\mathrm{~A} / \mathrm{V} \mathrm{m}$ & 0 & 58.1 & 4.72 & $\gamma(T)$ \\
\hline$\alpha$ & $\mathrm{V} / \mathrm{K}$ & 0 & 0 & 0 & $\alpha(T)$ \\
\hline $\mathcal{T}_{a d} \times 10^{6}$ & $\mathrm{~N} / \mathrm{m}^{2}$ & - & - & - & 60 \\
\hline
\end{tabular}

Table 1: Properties of the Peltier cell materials. For temperaturedependent properties see Eq. (1).

("hinged" vertical faces in Fig. 1). Also considering symmetry of the vertical $x_{2}=0$ plane, another half of the previous domain is eliminated (not visible in the plane of the figure). The hot and cold faces (top and bottom horizontal lines) are both considered hinged. Other mechanical boundary conditions (see Fig. 12) can easily be studied depending on the practical fastening of the cell, choice that strongly influences the level of stresses. The validity of the simplifications is verified in the next section.

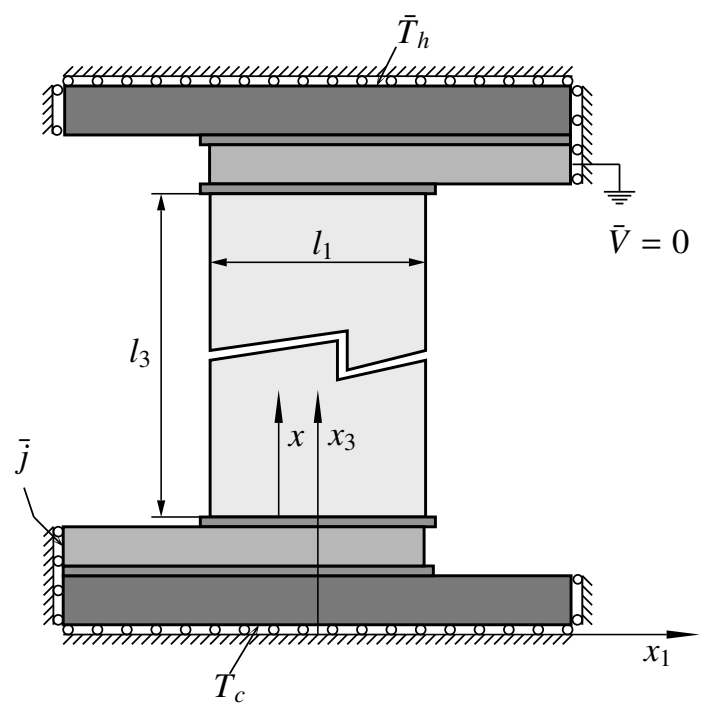

Figure 1: Scheme of the modelled half-thermocouple with boundary conditions. From higher to lower gray intensity: $\mathrm{Al}_{2} \mathrm{O}_{3}, \mathrm{SnPb}, \mathrm{Cu}$, $\mathrm{Bi}_{2} \mathrm{Te}_{3}$. Prescribed magnitudes denoted by an overbar.

The properties of the four materials listed in Table 1 are taken from [26] except the ones that depend on temperature, listed in Eq. (1) and taken from [27], and the ultimate stress from [28]. The mechanical $\lambda$ and $\mu$ are the isotropic Lamè parameters. Density is denoted by $\rho$, specific heat by $c_{p}$ and the thermal expansion coefficient by $\alpha_{T}$. The ultimate (admissible) stress $\mathcal{T}_{a d}$ is sometimes different for traction or compression; here we use a single value for lack of information. The Seebeck coefficient $\alpha$ is approximately linear but the electric and thermal conductivities $\gamma, \kappa$ are strongly quadratic from -55 to $+125^{\circ} \mathrm{C}$, the temperature range of interest.

$$
\begin{aligned}
& \alpha=1.988 \times 10^{-4}+3.353 \times 10^{-7} T+7.52 \times 10^{-10} T^{2} \\
& \kappa=1.663-3.58 \times 10^{-3} T+3.195 \times 10^{-5} T^{2} \\
& \gamma=1.096 \times 10^{5}-5.59 \times 10^{2} T+2.498 T^{2}
\end{aligned}
$$

Through the article and except for the experimental comparison, the cases will have the same geometry and boundary conditions. The TC works as a heat pump, with prescribed $\bar{T}_{h}=50{ }^{\circ} \mathrm{C}$ at the hot face and electric flux $\bar{j}$ (or equivalently electric intensity $\bar{I}=\bar{j} A$ for constant section), see Section 4. An efficient way to prescribe the electric flux in one of the $C u$ ends is with an 2D, massless interface element described in [20]. At least one of the nodes of the mesh must have the voltage $V$ prescribed, for this article to zero in the right of the upper $\mathrm{Cu}$. The rest of the surfaces are electrically and thermally isolated, including the cold face with variable $T_{c}$.

From Fig. 1, the TE cross-sectional area in plane $x_{1}$ $x_{2}$ is denoted by $A=l_{1} l_{2}$ where $l_{2}$ is the TE dimension perpendicular to the plane; for this cell, $l_{1}=l_{2}=0.001$ $\mathrm{m}$ and $l_{3}=0.0058 \mathrm{~m}$. For the calculation of stresses the reference temperature is taken as $T_{0}=20^{\circ} \mathrm{C}$.

A rectangular pulse of study with $P=2.5$ and duration $\Delta t_{p}=5 \mathrm{~s}$ will be considered, unless otherwise indicated. This pulse and its response in the cold face, see Fig. 2, is defined by the variables:

- $\bar{I} \equiv I_{o p}$, electric current applied up to $t_{s s 1}$ for maximum cooling through steady-state with resulting $T_{c} \equiv T_{c s s}$.

- $P$, Electric current amplification factor (gain) during pulse.

- $\bar{I}_{p}=P \bar{I}$, electric current applied at time $t_{s s 1}$ during pulse.

- $\Delta t_{p}=t_{p}-t_{s s 1}$, pulse duration, where $t_{s s 1}$ and $t_{p}$ are the initial and final pulse times.

- $T_{c m n}=T_{c s s}-\Delta T_{p}$ minimum temperature reached due to overcooling. 
- $\Delta T_{p}$ defined + , maximum transient temperature decrement from $T_{c s s}$ at instant $t_{m n}$.

- $\Delta T_{p p}$ defined + , maximum post-pulse temperature increment from $T_{c s s}$ at instant $t_{m x}$.

- $t_{s s 2}$, time when the steady-state is recovered after the pulse, then again $T_{c} \equiv T_{c s s}$.

In the simulation, $t_{s s 1}$ is defined as the earliest time for which the variables inside the TE (temperature, voltage, fluxes, stresses, etc.) are equal from one time step to those of the previous one, within a tolerance.
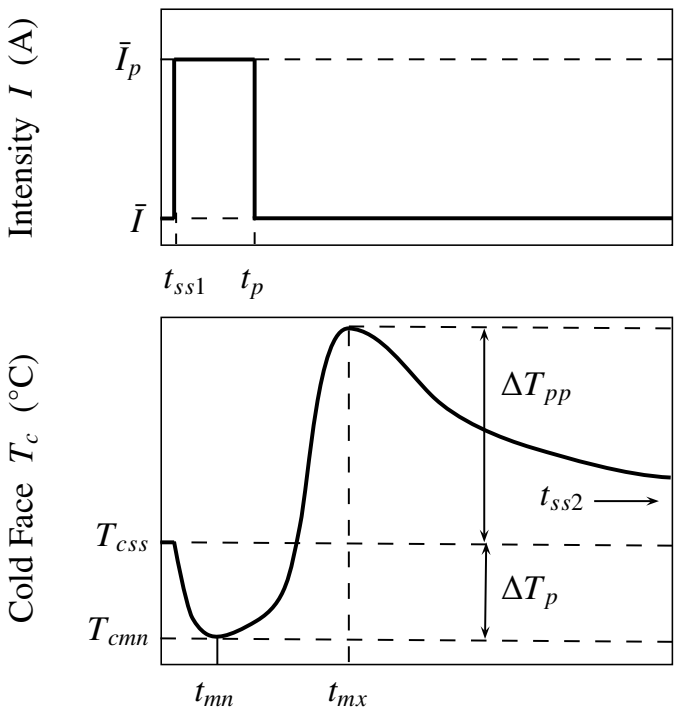

Time $t(\mathrm{~s})$

Figure 2: Main variables for pulsed thermoelectrics. Applied electric pulse (top) and measured temperature at cold face (bottom) vs. time.

\section{Finite Element formulation}

The transport (or constitutive, left) and equilibrium (right) equations that govern thermoelectric materials (TM) coupled with a mechanical field are:

$$
\begin{array}{ll}
\boldsymbol{j}=-\gamma \nabla V-\alpha \gamma \nabla T ; & \nabla \cdot \boldsymbol{j}=0 \\
\boldsymbol{q}=-\kappa \nabla T+\alpha T \boldsymbol{j} ; & \rho c_{p} \dot{T}= \\
& -\nabla \cdot \boldsymbol{q}-\boldsymbol{j} \cdot \nabla V-T_{0} \boldsymbol{\beta}: \dot{\boldsymbol{S}} \\
\boldsymbol{T}=\boldsymbol{C}: \boldsymbol{S}-\boldsymbol{\beta}\left(T-T_{0}\right) ; & \rho \ddot{\boldsymbol{u}}=\nabla \cdot \boldsymbol{T}
\end{array}
$$

where $\boldsymbol{q}$ and $\boldsymbol{j}$ are the vectorial thermal and electric fluxes and $\boldsymbol{C}$ the stiffness tensor. The thermal expansion tensor is denoted by $\boldsymbol{\beta}=\boldsymbol{C}: \boldsymbol{\alpha}_{T}$, where $\boldsymbol{\alpha}_{T}=$ $\left\{\alpha_{T}, \alpha_{T}, \alpha_{T}, 0,0,0\right\}^{\top}$ is a Voigt notation vector that holds for isotropic materials. The stress and strain tensors are usually denoted by $\boldsymbol{T}$ and $\boldsymbol{S}$ in electro-mechanical models to avoid notation confusion (viz. [29]). The equations are taken from [17] (with the addition of the thermal inertial term) and the mechanical ones from [21].

These partial differential equations can be discretized following the Galerkin method (viz. [30] for details), to give a specially developed FE algorithm. Five degrees-of-freedom (dof) are required to study elastothermoelectric couplings in three-dimensions (3D): three displacements, temperature and voltage. From [21] (without magnetic field), the assembled "stiffness" matrix is:

$$
\left[\begin{array}{ccc}
c_{1} \mathcal{K}^{U U}+c_{3} \mathcal{M}^{U U} & c_{1} \mathcal{K}^{U T} & 0 \\
c_{2} \boldsymbol{C}^{T U} & c_{1} \mathcal{K}^{T T}+c_{2} C^{T T} & c_{1} \mathcal{K}^{T V} \\
\boldsymbol{0} & c_{1} \mathcal{K}^{V T} & c_{1} \mathcal{K}^{V V}
\end{array}\right]
$$

The three diagonal submatrices represent the direct interactions for the three fields of study: mechanical, thermal and electric. No mechanical damping effects are considered for the targeted cases although they could easily be added (but the solving would be more complicated); the mass $\mathcal{M}^{U U}$ is included for mechanical inertial forces. The thermal field considers the capacity matrix $\boldsymbol{C}^{T T}$ to simulate thermal dynamics and $\boldsymbol{\theta}$ can be a $3 \times 1$ or a $1 \times 3$ zero vector.

The thermoelastic interactions are not symmetric since the coupling from thermal to mechanical fields is, in principle, considered one-way. That is, the temperature difference produces strains through coefficients of thermal expansion included in $\mathcal{K}^{U T}$, but these strains do not statically dissipate energy due to the hypothesis of linear elasticity. To correct this simplified oneway coupling, Biot's principle considers the coupling matrix $C^{T U}$, that introduces dissipation due to mechanical vibrations. This term is proportional to the rate of strain, relatively small in the cases of the current work. The thermoelectric interactions are represented by $\mathcal{K}^{T V} \neq \mathcal{K}^{V T}{ }^{\top}$ due to Peltier, Seebeck and Thomson effects.

The complete physical interpretation and mathematical development of these submatrices, as well as their FE implementation including the integration parameters $c_{1}, c_{2}, c_{3}$ (viz. [30]) are described in [20] in detail and will not be repeated here. The elements are isoparametric hexahedral, and given the particular geometry of the 
TEs the mesh is structured.

As a first step and for a steady-state generic case, in Fig. 3 a comparison between deformed FE meshes is presented; in the left, three thermocouples in series, in the right, half of one thermocouple taking advantage of the symmetries. Even for a relatively coarse mesh the displacements are virtually identical in both meshes; temperature, voltage and related fluxes are completely equal, and Tresca equivalent stresses vary only $2.5 \%$. For this equivalent stress we use the expression $\mathcal{T}_{t r}=$ $\left|\mathcal{T}_{I}-\mathcal{T}_{I I I}\right|$, which has to be smaller than $\mathcal{T}_{\text {ad }}$ and where the roman subindices are the first and third principal stresses. This failure criteria gives very similar results to those from the references listed in Section 1 using Von Mises.

The solder is modeled with only one element along $x_{3}$ and with lateral burrs, to represent real weldings and to take into account the stress relieve that they can introduce (see Section 6).

The scalar fields of temperature and voltage are easy to capture with a few linear elements, but to do so for the thermal and electrical fluxes and specially the stresses with some precision is much more demanding. These stresses are mostly induced by the different expansion coefficients and tend to strongly concentrate at corners that join two different materials. Even for refined meshes, the linear eight-node element is not very suitable to capture these concentrations, therefore the quadratic 27-node is chosen. The final mesh includes 376 elements and 5280 nodes, with a total of 19267 active dof, enough to reasonably capture all magnitudes. The solution of this mesh takes $4000 \mathrm{~s}$ in an Intel Core i7-4810 MQ running at $2.8 \mathrm{GHz}$ for the typical case of VP described in Section 2. The TE itself has 160 elements with all 9225 active dof.

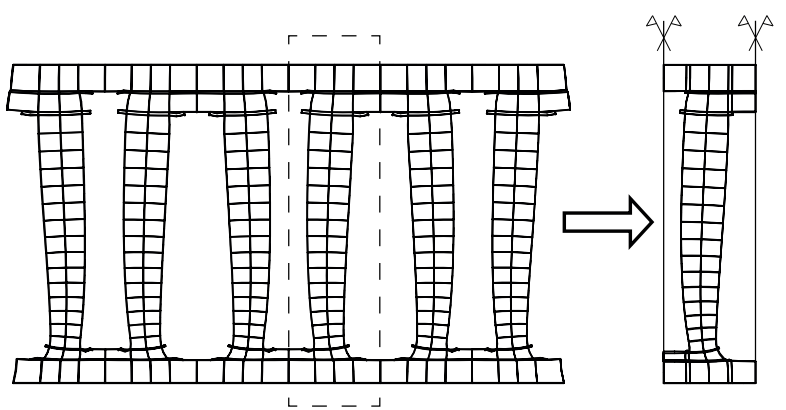

Figure 3: Comparison of the deformed configuration between three thermocouples in series and without lateral restriction (left) with half of a single thermocouple with repetition lateral restriction (right).

The prescribed electric intensity described in the next section is introduced in the symmetric (for repetitions) left vertical side of $C u$ in Fig. 1 using the mentioned interface 2D finite element.

A rather small time increment $\Delta t=0.05 \mathrm{~s}$ is necessary for the integration of the dynamic equation. The reason for this fine discretization is the study of a rectangular electric pulse, that in the instant of application requires the prescription of an intensity step as small as possible. Small roundoffs at that dynamic iteration drive significant variations in the calculation of $T_{c s s}$. The integration of the nonlinearities is solved without problems thanks to the non-symmetric iterative solvers of FEAP.

\section{Steady-state optimal electric intensity}

From [27] and CP, the simplified balance of energy in the cold face of a 1D TE with longitudinal coordinate $x$ (origin at the bottom side close to the cold face, see Fig. 1) and in steady-sate, is defined by the Peltier, Fourier and Joule effects, respectively:

$$
Q_{c}=\alpha \bar{I} T_{c}-\frac{\kappa A}{l_{3}}\left(\bar{T}_{h}-T_{c}\right)-\frac{l_{3}}{2 \gamma A} \bar{I}^{2}
$$

where $Q_{c}$ is the cooling thermal power extracted from the cold face. While Joule's term depends quadratically on the intensity, Peltier does linearly, therefore, an optimal intensity $I_{o p}$ that maximizes this power exists. Its expression can be calculated by derivation to give $I_{o p}=A \alpha \gamma T_{c} / l_{3}$, but this result depends on the unknown $T_{c}$. To reach a computable maximum of $\bar{T}_{h}-T_{c}$ for steady-state $Q_{c}=0$ is imposed and the previous equality is inserted into Eq. (4) giving:

$$
T_{c m n}=\frac{-1+\sqrt{1+2 \bar{T}_{h} Z}}{Z}
$$

where the figure of merit is $Z=\alpha^{2} \gamma / \kappa$. Note that this minimum only depends on $\bar{T}_{h}$ and material properties, not on geometry. Inserting now Eq. (5) in the expression of $I_{o p}$, the intensity to prescribe is:

$$
I_{o p} \equiv \bar{I}=\frac{A \kappa}{l_{3} \alpha}\left(-1+\sqrt{1+2 \bar{T}_{h} Z}\right)
$$

The equation for the power heat $Q_{h}$ given to the hot face is the same as that of $Q_{c}$, except that the third term is positive (conduction adds power from Joule to this face).

Even if $\mathrm{CP}$ are considered, $T\left(x_{3}\right)$ varies inside the TE and it is not clear how to choose the properties $\alpha, \gamma, \kappa$ for Eq. (6). Therefore, a simple iterative method to calculate the optimal intensity with $\mathrm{CP}$ and for fixed $l_{3}$ and 
$\bar{T}_{h}$ is developed. As a first iteration, $\bar{I}$ for an estimated constant average $T_{a v}$ is found with Eqs. (1) and (6) and $T_{c}$ with Eq. (5). A more precise calculation with again constant $T_{a v}=\left(\bar{T}_{h}+T_{c}\right) / 2$ can be now made and the process is repeated until the intensity converges.

The situation is more complicated for VP since is $Z$ is also variable along the TE and Eq. (6) is not anymore a good approximation. Further refinement can be found bisecting the results of several runs of the FE code under VP (FEVP) and forking from the previous $\bar{I}$ to find the accurate minimum $T_{c}$, see Fig. 7 top. But this process could add a substantial calculation cost to situations in which many runs are necessary, e.g. optimization. In any case and if necessary, this correction fixes in part the previous simplifications.

For the conditions given in Section 2 and a first estimation $T_{a v}=35{ }^{\circ} \mathrm{C}$ the formulae gives $\bar{I}=0.83 \mathrm{~A}$ with $T_{c m n}=-29.09{ }^{\circ} \mathrm{C}$ and therefore a rather different $T_{a v}=10.45^{\circ} \mathrm{C}$. After just four iterations $\bar{I}=0.888 \mathrm{~A}$ with $T_{c m n}=-28.46{ }^{\circ} \mathrm{C}$ are obtained; this is the intensity prescribed through most of the paper with CP. If the additional FEVP bisection is used, the final result is $\bar{I}=$ 0.917 A with $T_{c m n}=-32.04{ }^{\circ} \mathrm{C}$, about a $11 \%$ increase from that of $\mathrm{CP}$; this is the intensity prescribed through most of the paper for VP. Note that although the difference between the last two intensities is small, that of the minimum $T_{c}$ (the most important parameter) is not.

\section{Validations}

\subsection{Analytical validation}

In this subsection a simple semi-analytical model for a single TE subjected to a pulse will be developed, under several simplifying assumptions: i) $1 \mathrm{D}$ electric flow $j$, ii) temperature independent properties, $\mathrm{CP}$, iii) mechanical displacements decoupled from the other dof's and iv) $j$ decoupled from the thermal field, in contradiction with the first of Eqs. (2).

The geometry is prismatic as in an isolated TE from Fig. 1 with longitudinal coordinate $x$. Combining Eqs. (2) and taking into account the assumptions:

$$
\rho c_{p} \frac{\partial T}{\partial t}=\kappa \frac{\partial^{2} T}{\partial x^{2}}+\frac{j^{2}}{\gamma}
$$

with boundary conditions

$$
T\left(l_{3}, t\right)=\bar{T}_{h} ; \quad \frac{\partial T(0, t)}{\partial x}=\frac{\alpha \bar{j}}{\kappa} T(0, t)
$$

The first condition is a prescription of temperature on the top side; the second comes from the left middle expression of Eqs. (2) considering that on $x=0$ the heat flux is isolated $q(0)=0$. The initial condition assumes constant temperature equal to that of the top side, $T(x, 0)=\bar{T}_{h}$.

Due to the dynamic nature of the problem, the Laplace transform is applied to Eq. (7) to convert a partial differential equation into an ordinary one. Defining the transformed temperature as $T_{L}(x, s) \equiv \mathscr{L}\{T(x, t)\}$ :

$$
\begin{aligned}
& \rho c_{p}\left[s T_{L}(x, s)-T(x, 0)\right]=\kappa \frac{\partial^{2} T_{L}}{\partial x^{2}}+\frac{\mathscr{L}\left\{\bar{I}^{2}\right\}}{A^{2} \gamma} \\
& T_{L}\left(l_{3}, s\right)=\frac{\bar{T}_{h}}{s} ; \quad \frac{\partial T_{L}(0, s)}{\partial x}=\frac{\alpha T_{L}(0, s) \bar{I}}{A \kappa}
\end{aligned}
$$

The intensity $\bar{I}$ has been assumed picewise constant in the three stretches of Fig. 2 top, that is, the equation must be solved for three time intervals. For the first interval a double integration produces two constants that are solved with the boundary conditions from Eq. (9), resulting in:

$$
\begin{array}{ll}
T_{L}(x, s) & =\frac{\bar{T}_{h}}{s}+\frac{\bar{j}}{s^{2} \gamma \rho c_{p}}\left(\bar{j}-\frac{f_{1}+f_{2}+f_{3}}{f_{4}}\right) \\
f_{1} & \equiv \bar{j}^{2} \alpha \sinh (\Lambda x) \\
f_{2} & \equiv \alpha\left(\bar{j}^{2}+\bar{T}_{h} \kappa \gamma \Lambda^{2}\right) \sinh \left(\Lambda\left(l_{3}-x\right)\right) \\
f_{3} & \equiv \bar{j} \kappa \Lambda \cosh (\Lambda x) \\
f_{4} & \equiv \bar{j} \alpha \sinh \left(\Lambda l_{3}\right)+\Lambda \kappa \cosh \left(\Lambda l_{3}\right)
\end{array}
$$

where $\Lambda \equiv \sqrt{s / a}$ and $a=\kappa / \rho c_{p}$ is the thermal diffusivity. This expression is too complicated to directly be anti-transformed. Instead, the GWR algorithm [31] that depends on only one parameter (precision of 16 significant digits) is used.

Once the first stretch is solved, the initial condition for the second is easily calculated particularizing the anti-transformed numerical solution to its final time $t_{s s 1}$. The solution is fitted into a quadratic polynomial so that the initial condition becomes $T\left(x, t_{s s 1}\right)=b_{0}+b_{1} x+b_{2} x^{2}$, reassigning $t_{s s 1}=0$. Alternatively and for this pulse, these polynomial terms can be directly deduced from the direct integration of Eq. (7) of the first stretch equating to zero the dynamic term:

$$
T(x, 0)=\bar{T}_{h}+\left(\frac{\bar{j}^{2}}{2 \gamma \kappa} l_{3}-\frac{\bar{T}_{h}-T_{c}}{l_{3}}\right) x-\frac{\bar{j}^{2}}{2 \gamma \kappa} x^{2}
$$

where $T_{c}$ is given by Eq. (5). Following the same steps, an expression similar to Eq. (10), although with more terms, is obtained for the second stretch.

For the third stretch starting at time $t_{p}$ the same procedure is used again, obtaining another expression function of new polynomial coefficients. 
The complete temperature distribution will be studied in Fig. 5, but some preliminary results can be given here. The material properties are defined in Table 1, with geometry and boundary conditions from Sections 2 and 4. It is obvious that an unit increment of $P$ should translate into a certain increment of $\Delta T_{p}$, but the improvement substantially reduces as $P$ reaches high values. The reduction is due to the quadratic Joule heat generation that forces the additional overcooling to tend to zero. The continuity of the present analytical model is convenient for the study of this phenomenon since $T_{c}$ can be calculated with precision with an algorithm of minimum search based on progressive time step reduction. With the FE method, the minimum $T_{c}$ (and then $\Delta T_{p}$ ) can easily be overshoot since each value close to it comes from a different run.

With this algorithm and integer values of $P \geq 1$, Fig. 4 is plotted. The ordinate is the overcooling increment $\left.\Delta T_{c m n}\right|_{i+1}=\left.T_{c m n}\right|_{i+1}-\left.T_{c m n}\right|_{i}$ when the abscissa $P$ is incremented one unit from integers $i$ to $i+1$. Up to pulses $P<3$, the absolute $T_{c m n}$ increases almost linearly and with a high slope. For larger pulses the Joule effect strongly reduces this increment, and for $P>15$ asymptotically tends to zero very fast. For instance, raising $P$ from 1 to 2 produces an increment of $11.9^{\circ} \mathrm{C}$ while raising it from 5 to 6 increases only $1.5^{\circ} \mathrm{C}$. In any case, very high gains are unrealistic as they unacceptably accumulate overheating and the induced stresses would exceed the admissible one, see Section 6.

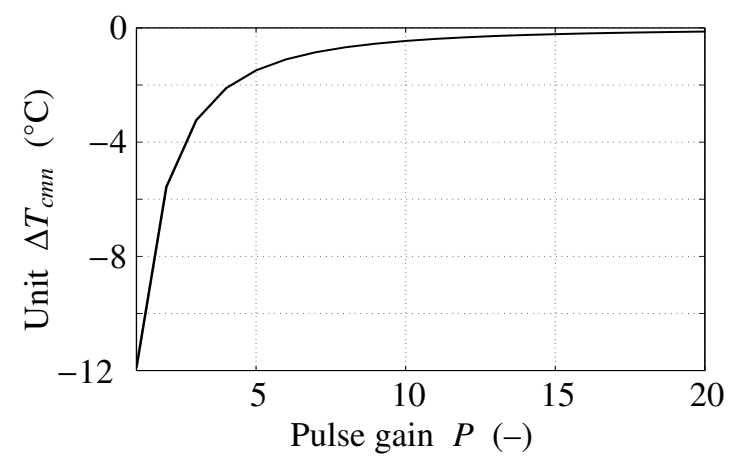

Figure 4: Cold face temperature overcooling increase due to a unit increment of pulse gain, calculated with analytical solution.

As a first validation, a comparison is shown in Fig. 5 from a few instants before the pulse application to $50 \mathrm{~s}$ after the maximum overheating is reached. The pulse lasts $\Delta t_{p}=4.8 \mathrm{~s}$, the hot face is at $\bar{T}_{h}=-6.4{ }^{\circ} \mathrm{C}$ and the corresponding intensity is $\bar{I}=0.675 \mathrm{~A}$. For the calculations, $\bar{T}_{h}$ has been slightly reduced to produce the same $T_{c s s}=-56{ }^{\circ} \mathrm{C}$ for the: i) analytical 1D model, ii) the
3D FE with CP (FECP) and iii) experimental from [4] (see next subsection). The fundamental properties $\alpha, \gamma$, $\kappa$ are indirectly calculated from the reference using simple closed-form expressions and values of $Z T_{c s s}, a$ and $\bar{I}$, maintaining $\rho c_{p}$ from Table 1 . Other trio combination from additional values give different fundamental properties, adding to the uncertainty of the experiment basic conditions. The VP from Eqs. (1) cannot be calculated since they require nine parameters.

The distributions of the analytical and the FE models are very similar along the first and second stretches, but in the post-pulse the former predicts a few degrees less, primarily due to assumption iv) described before. Similarly, there is also a difference of $3{ }^{\circ} \mathrm{C}$ for $\Delta T_{p}$ between both models and the experiments, probably due to the uncertainty in the values of the properties that are very sensitive for the overcooling calculation. One of the conclusions of this comparison is that non-linearities, 3D geometry, the Biot term etc. do make a difference for $\Delta T_{p p}$ but not much for $\Delta T_{p}$ or distributions before the corresponding time.

The numerical FE calculations from instant zero to steady-state require a large amount of computational time: the guessed initial condition (room temperature for instance) is in general very different from the permanent quadratic distribution. Therefore many dynamic iterations are necessary for the convergence to an acceptable steady-state. The convergence is much slower with FEVP due to the strong additional non linearities. To avoid the expense of this computational time, that does not add any information to the pulse study, a simple static case is run; the calculated static distributions constitutes the initial conditions of the proper dynamic run, choosing the origin as $t_{s s 1}=0$. At a long time after the removal of the pulse (around $t_{s s 2}>80 \mathrm{~s}$ ), all curves of Fig. 5 tend again to the cold face temperature $T_{c s s}$.

\subsection{Experimental validations}

One of the interesting contributions of the cited experimental article is that proposes general expressions independent of materials and boundary conditions. In particular, the overcooling measurements are repeated for several conditions, fitting the empirical relationships:

$$
\Delta T_{p} \approx \frac{1-\mathrm{e}^{(1-P)}}{4}\left(\bar{T}_{h}-T_{c s s}\right) ; \quad t_{m n} \approx \frac{l_{3}^{2}}{4 a} \frac{1}{(1+P)^{2}}
$$

To check the validity of these general expressions, the material properties are taken not from [4] but from Table 1 , and boundary conditions from Section 4 . Due to symmetry, the mesh simulates a single TE with the 


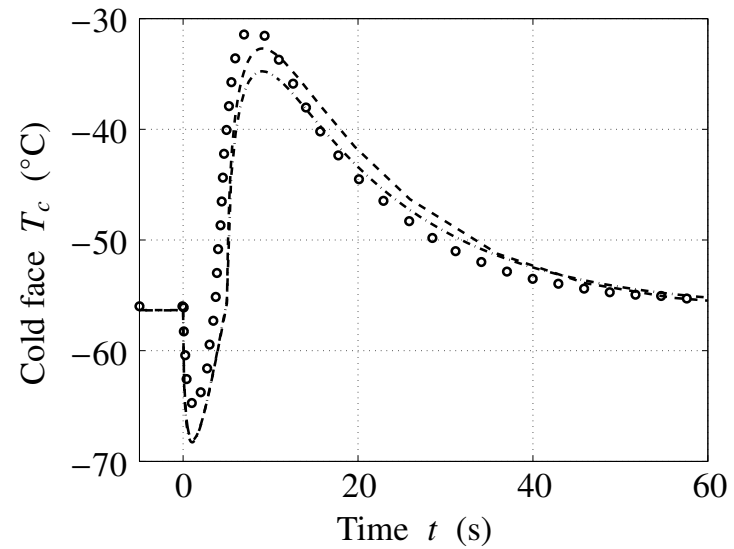

Figure 5: Cold face temperature transient distribution. Analytical (dash-dot), finite element results with constant properties (dashed) and experimental from [4] (circles).

same dimensions as before, and only two (top and bottom) cooper foils of thickness $l_{C u}=35 \times 10^{-6} \mathrm{~m}$ as depicted in the reference.

Figure 6 shows the distribution of Eq. (12) and the FECP and FEVP solutions, the last two calculated with $P$ increments of 0.25 . The reference declares that measured values $P>3$ are not reliable due to possible influence of aliasing and other reading errors. In the case of $\Delta T_{p}$ (left ordinate), the agreement is very good for $P \leq$ 3 (maximum $11 \%$ error) and starts diverging for greater values, with a $17 \%$ difference for $P=4$. The FECP result (dashed line) is remarkably very similar to the linear model described in [4] (not shown in the figure).

Note that with FEVP the predicted overcooling should be higher than with FECP since: i) Joule heat is reduced by Thomson effect proportional to $\mathrm{d} \alpha / \mathrm{d} T$ and ii) the conductive Fourier heat decreases with increasing $T$ by the quadratic dependency of $\kappa$ on it given by (1) (in the range of the cases shown here). The curve for FECP predicts a higher $\Delta T_{p}$ but the reason is its lower $\left|T_{c s s}\right|$, that is, the total overcooling itself is larger for FEVP but not the difference $\Delta T_{p}$.

In the same figure and with right ordinate, the time $t_{m n}$ to reach the maximum $\Delta T_{p}$ (minimum $T$, see Fig. 1) is plotted. The almost perfect coincidence in most of the range of FECP with the experimental regression of Eq. (12) right does not have physical meaning, given the aforementioned uncertainties in the properties. It can be appreciated that the experimental regression is wrong for low $P$ 's: the value of $t_{m n}$ should tend to $\infty$ for $P \approx$ 1 (no pulse implies no overcooling, see again Fig. 1) instead of the given $5 \mathrm{~s}$. As expected, both FECP and FEVP simulations tend to a very large value for this $P \approx$
1. On the contrary, $t_{m n}$ correctly tends to a small value for all curves when $P$ becomes very large.

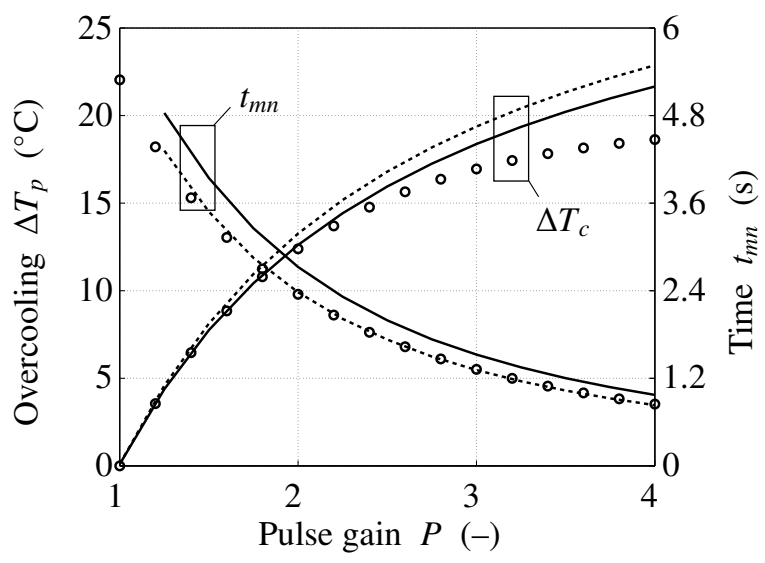

Figure 6: Maximum transient temperature overcooling at cold face (left ordinate). Time to reach this maximum (right ordinate). Finite element results for constant (dashed), variable material properties (solid) and regression of experiments [4] (circles).

\section{Thermoelectric results}

The first result is related to the developments from Section 4. In Fig. 7, $T_{c m n}$ (top) and the maximum Tresca stresses at any point of the TE (bottom) are plotted versus the prescribed intensity, both at steady-state.

In the top figure, the minimums of the FECP and FEVP curves are equal to the iterated $I_{o p}$ from Eq. (6) and the bisected, respectively. Under this intensity, Peltier supersedes the other effects, For other values of $I,\left|T_{c}\right|$ is smaller (less overcooling), with slow decreases for low values but very fast for high ones. The trend of both curves is very similar around $I_{o p}$, but for $I>1.4 I_{o p}$ these curves strongly diverge. It is important to note that with CP the $T_{a v}$ corresponding to $I_{o p}$ is used for all intensities; in the current range of temperatures the differences are not important.

About thermal fluxes, for intensities lower than $I_{o p}$ both Peltier and Joule are small and conduction is prevalent. For higher intensities Joule becomes prevalent, resulting in TE higher temperature distributions than the corresponding to the optimal.

Logically the stresses increase with $I$ since the maximum $T$ inside the TE also increases (Fig. 8 top), interestingly enough very slowly for intensities up to $1.5 I_{o p}$ but with increasing slope for higher values. An intensity $1.8 I_{o p}$ already produces a stress higher than the admissible; therefore, the optimal $I_{o p}$ is not only a value useful to maximize overcooling but also a limit to not over-stress the TE. Elastic studies must be done for PT 
designs since TM present, specially under tension, low mechanical strengths, [34].

Strains and displacements are proportional to the difference of temperatures and the first derivative of these displacements proportional to stresses; at $I_{o p}$ the slope of $T_{c}$ changes sign and then the curvature of $\mathcal{T}_{t r}$ also changes from slightly convex to concave. Since for $I \leq 1.4 \mathrm{~A}$ the slope of $T_{c m n}$ is small so are the stresses; where the $T_{c m n}$ slope becomes constant $(I \geq 1.4 \mathrm{~A})$ the curvature of the stress becomes $\infty$ (straight line) and with a strong increment.

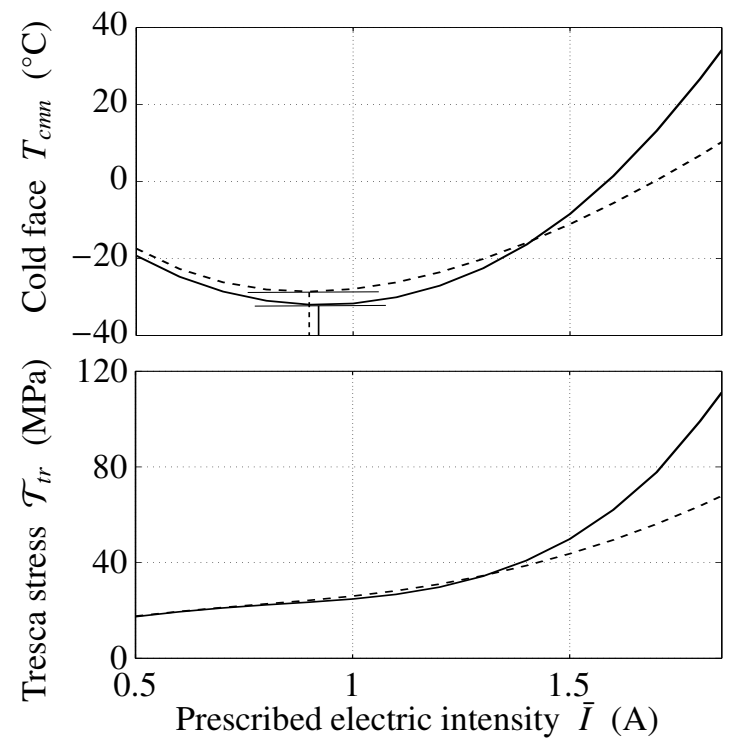

Figure 7: Steady-state cold face temperature (top) and maximum thermoelement Tresca stresses (bottom) vs. prescribed intensity. Finite element with variable (solid) and with constant properties (dashed line).

Figure 8 plots the temperature evolution along the TE length calculated with FEVP. The top figure is for four representative instants during the pulse with the conditions of Section 2 . At the stationary $t_{s s 1} \equiv 0 \mathrm{~s}$ the distribution is slightly quadratic from an overcooling $T_{c m n}$ $=-32{ }^{\circ} \mathrm{C}$ (TE bottom $x_{3} / l_{3}=0$, close to cold face) to a value similar to the prescribed $\bar{T}_{h}=50{ }^{\circ} \mathrm{C}$ (TE top $x_{3} / l_{3}$ $=1$, close to hot face). At $t_{m n}=4 \mathrm{~s}$, the pulse forces the maximum (in absolute value) $T_{c m n}=-40{ }^{\circ} \mathrm{C}$ and the curve changes sign close to the origin, point in which Joule overcomes Peltier. On $x_{3} / l_{3}=0.7$ the maximum $T$ doubles and is $60 \%$ higher than $\bar{T}_{h}$; the temperature at the TE end is a significant $20 \%$ higher than $\bar{T}_{h}$.

After $1 \mathrm{~s}$ the pulse is removed and $\Delta T_{p}$ remains almost the same although most of the distribution is slightly higher. For a longer time $\left|T_{c}\right|$ keeps decreasing due to the lowering of Peltier and the delayed arrival of Joule to the cold face from the rest of the TE. At $t \approx 20$ s the maximum overheating $T_{c}-20{ }^{\circ} \mathrm{C}$ is reached, with a relaxed distribution and now with a top temperature very similar to $\bar{T}_{h}$ at $x_{3} / l_{3}=1$. As a conclusion, due to Joule the temperature inside the TE can be much higher than $\bar{T}_{h}$, fact that has consequences for the calculation of stresses.

In Fig. 8 bottom the same distributions are plotted, but now for time $t_{m n}$ and integer values $1 \leq P \leq 4$. The curve for $P=1$ is obviously equal to that of steady-state of the top figure. Note that for $P \geq 2$ the increment of $\left|T_{c m n}\right|$ is relatively small in the plot range, trend already shown in the asymptotic Fig. 4. The equality is even more evident here since the whole FE mesh is used in these calculations and more materials are present (see next Fig. 9). For $P=4$ a compelling maximum of $T=$ $120{ }^{\circ} \mathrm{C}$ is reached.

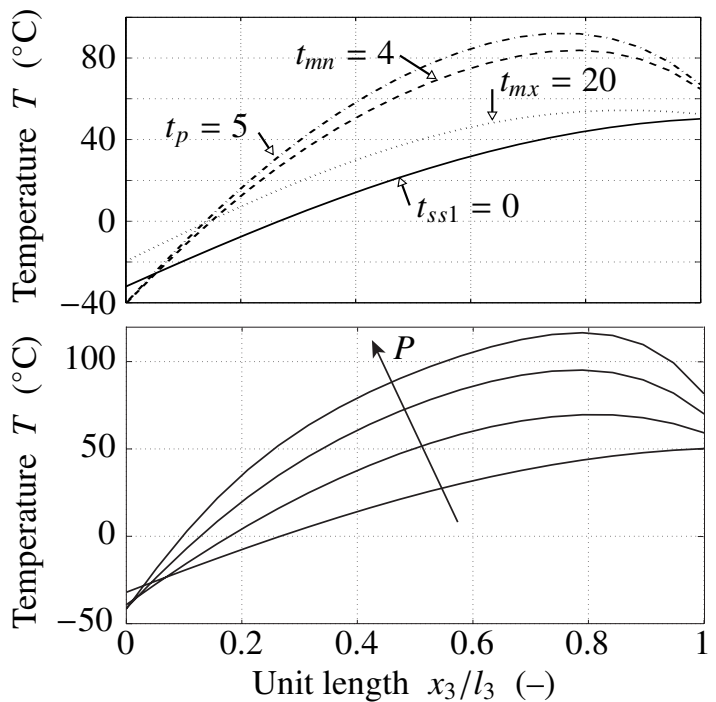

Figure 8: Top: temperature along thermoelement for: steady-state, maximum overcooling, pulse removal, maximum overheating, fixed $P=2.5$. Bottom: idem for integer gains $1 \leq P \leq 4$ at maximum overcooling with variable $t_{m n}$. Finite element and variable properties.

In Section 2, it was mentioned that consideration of the non-thermoelectric materials in the simulation was relevant for thermal dynamics. Figure 9 and only with FEVP shows the overcooling process for a mesh with only TE (thin line) and another mesh with all materials from Fig. 1 as in commercial devices (thick line). It is obvious that the absence of some thermal inertias $\rho c_{p}$ produces different dynamics and several points should be highlighted. For the thin line (only TE mesh):

- $T_{c s s}$ is slightly lower due to the small voltage drop produced by $\mathrm{Cu}$ and $\mathrm{Sn}_{2} \mathrm{~Pb}_{3}$ when present.

- The cooling and heating curves present higher slopes (faster dynamics) due to the absence of thermal inertias from additional masses. 
- For the same reason, $t_{m n}$ and $t_{m x}$ are smaller, and both $\Delta T_{p}, \Delta T_{p p}$ substantially higher.

- The return to steady-state is also faster, that is, $t_{s s 2}$ is much smaller.

The conclusions are that all materials (including $\mathrm{Al}_{2} \mathrm{O}_{3}$ ) are important not only for the calculation of stresses but also for the dynamics of the thermal field: a duplication of $\Delta T_{p}$ can be appreciated between the two meshes, with an obvious large error. Also, that the thinner the non-TE materials the better, and that masses attached to the cold face should not be very large since then the overcooling would be canceled. For other boundary conditions or pulse gains and durations, thermal breakdown can occur inside the TE.

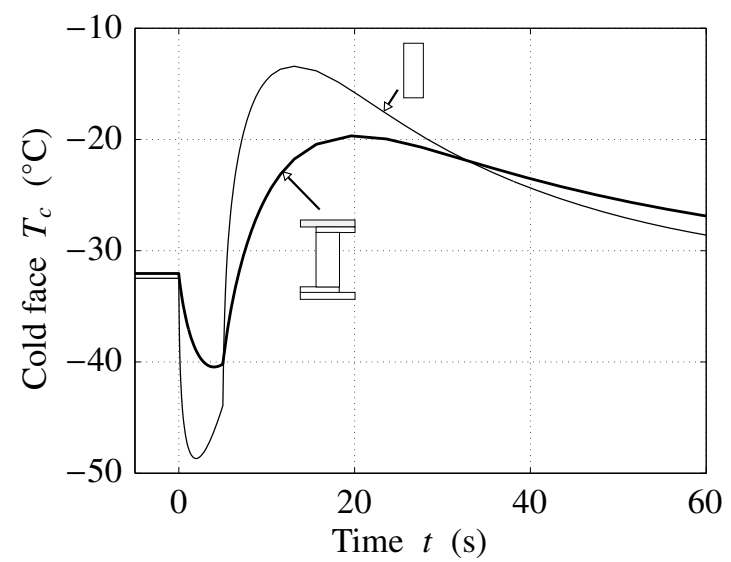

Figure 9: Non-thermoelectric materials transient influence on thermal inertia. Finite element with variable properties; thin line mesh with only thermoelement, thick line with complete mesh as in Fig. 1.

\section{Mechanical results}

A complete mechanical stress analysis of the TC is presented in this section. As will be shown, in normal dynamic conditions these stresses can reach relatively high values in the TE and very high values in the other materials. The stresses are specially relevant in $\mathrm{Al}_{2} \mathrm{O}_{3}$ with the highest rigidity but also in $\mathrm{Cu}$ and $\mathrm{SnPb}$ due to the $\alpha_{T}$ mismatch. A precise stress analysis would require the consideration of plasticity, relaxation and fatigue, but these effects are out of the scope of this article and therefore the stresses will be studied inside the TE, where it is assumed that the elastic range is maintained.

The electrical magnitudes determine the temperature distribution inside the TE. Then, the higher the difference of $T$ at a point with respect to $T_{0}$ and the closer this point to movement restrictions, the larger the stresses will be. The position of the maximum stresses will be in the unions between solder and TE and that of the minimum its vertical center. However, the maximum deformations appear close to this center, since $T$ is higher due to Joule and no restrictions are present. With respect to time, the overall maximum stresses will happen at the end of the pulse $t_{p}$ due to the cumulative temperature increase. Once the pulse is removed at this $t_{p}$, the temperatures quickly drop (see Fig. 8 top) and so do the stresses.

To better understand the stress causes and distributions, in Fig. 10 the deformed configuration at two representative instants is drawn in two planes: sideways $x_{2}-x_{3}$ and front $x_{1}-x_{3}$. Gray lines represent the initial configurations and black lines the deformed ones. The study of strains/stresses in 3D is relevant since the isotropic $\alpha_{T}$ has equal values in the three directions.

Firstly and from Table 1 it is worth to mention that $C u$ and TM are fairly compatible in thermal expansion, but $\mathrm{Cu}$ and $\mathrm{Al}_{2} \mathrm{O}_{3}$ are not; due to the geometry of Fig. 1 this incompatibility will be relevant mostly in the transversal $x_{1}, x_{2}$ directions. The $S n P b$ is not important in this aspect due to its very small thickness. The low $\alpha_{T}$ of the $\mathrm{Al}_{2} \mathrm{O}_{3}$ and its double and mutually perpendicular mechanical restriction almost freezes its movement. Note that at the union of any two materials the different "free" elongations are automatically made compatible by the continuity of the FE mesh, resulting in traction stresses for one and compression for the other.

From the distributions of Fig. 8 top, it can be appreciated that the TE will tend to expand in its top half $T\left(x_{3}\right)>T_{0}$ and to contract in its bottom during steadystate (viz. left pair of Fig. 10); at pulse removal $t_{p}, 80 \%$ of the TE is in expansion (right pair). As expected, the general displacements are larger in the right pair, specially in the zone where $T\left(x_{3}\right)$ is highest.

The mechanical restrictions of symmetric repetition applied to the $x_{1}$-sides "hinge" the two assemblies $C u$ $\mathrm{Al}_{2} \mathrm{O}_{3}$ at the two extremes of $\mathrm{Al}_{2} \mathrm{O}_{3}$ but of only one of $\mathrm{Cu}$. Both $\mathrm{Cu}$ strips tend to horizontally move towards left or right with a different amount than that of the attached TE and therefore the assemblies tend to rotate, the top one clockwise and the bottom one counterclockwise. The vertical $x_{3}$ restrictions at the external hot and cold faces mostly prevent these rotations, creating compressions in the $x_{1}, x_{2}$ free directions of the $C u$ corners of the top assembly and tractions in the bottom one. Figure 10 is a detailed version of Fig. 3 and highlights the presence of these compression/tractions at the corners. The combination of vertical and horizontal mechanical restrictions creates a type of "slide" boundary conditions at the ends of the TE, resulting in a middle line 
movement similar to that of two-plane bending but with swollen barrel-shape deformation. This slide condition is only partial since it allows a small rotation and displacement in $x_{1}$ and $x_{2}$.
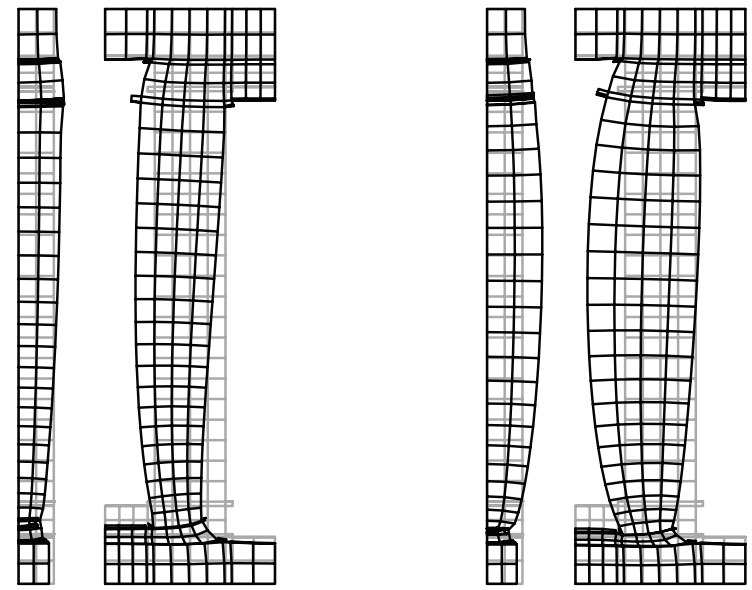

Figure 10: Deformation $\times 400$ with finite elements and variable properties. Left pair at steady-state, right pair at pulse removal $t_{m n}=5$ s. See Fig. 1 for boundary conditions and Fig. 8 top for temperature distributions in thermoelement.

Figure 11 shows the stresses in half of the TE: the visible vertical side is the symmetric, "cut" one. The rows are for three representative instants: steady-state $t_{c s s} \equiv 0$, pulse removal $t_{p}$ and maximum overheating $t_{m x}$, see Fig. 2. Five components (axes in the top left figure) are plotted in columns, the sixth $\mathcal{T}_{x_{1} x_{2}}$ is almost zero since in planes of constant $x_{3}$ there are no thermal expansion mismatches. The units in the scales are MPa, clear grays represent minimums and dark ones maximums.

The normal components $\mathcal{T}_{x_{1}}, \mathcal{T}_{x_{2}}$ and $\mathcal{T}_{x_{3}}$ concentrate at the bottom, due to the significant movement restriction and in particular to the mentioned maximum temperature difference. There is a similar concentration at the top but of lower value and extension. The shear components $\mathcal{T}_{x_{i} x_{j}}, i \neq j$, also appear close to restrictions, where the distortion from a rectangle to a parallelepiped element in any plane is important.

There are two reasons for the appearance of the normal $\mathcal{T}_{x_{1}}$. First, due to the restriction that $\mathrm{Al}_{2} \mathrm{O}_{3}$ exerts on $\mathrm{Cu}$ : close to the cold face and in $x_{1}-x_{3}$, the bottom surface of $C u$ is almost fixed, while its the top surface right edge moves left. Second, for the superimposition of a 3D local bending with tractions in one side and compressions in the other. The final result is shown in the first column, with tractions shifted from the left edge to the center and compressions around the right edge. The tractions do not reach the free side of constant $x_{2}$ to fulfill the boundary conditions.

Note that the repetition boundary condition increases this component; in reality the horizontal $x_{1}$ movement caused by the thermal expansion will be only completely restricted in some of the central TC. But the influence will be present mostly in the $\mathrm{Al}_{2} \mathrm{O}_{3}$ and $\mathrm{Cu}$ and nevertheless its consideration will give slightly conservative results.

For $\mathcal{T}_{x_{2}}$ the situation is similar although there is no external lateral restriction in the plane $x_{1}-x_{3}$ at $C u$; then, its $\alpha_{T}$ mismatch with $S n P b$ is relevant since it is the only restriction present. At the bottom, this component is of compression only. The signs of $\mathcal{T}_{x_{1}}, \mathcal{T}_{x_{2}}$ are reversed at the top face since the temperature is above $T_{0}$, see Fig. 8 top.

The plots of the other normal $\mathcal{T}_{x_{3}}$ respond to the restriction that the boundary slides exert on the TE ends and also the TE cumulative tendency to expand. The bending in the sideways plane obliges the maximum stress to be at the ends although the concentration is shifted due to the local rotation. Equal to $\mathcal{T}_{x_{1}}$, the maximum traction stress appears almost at the center of the TE bottom surface, although the compressive concentrates at the external edge due to the prevalence of bending in plane $x_{2}-x_{3}$.

The shear $\mathcal{T}_{x_{2} x_{3}}$ also appears mostly at the vertical TE ends, and it is zero in most of the TE center. It peaks in the free planes $x_{2}= \pm l_{2} / 2$ since they have more freedom to distort. In the non-simulated half (symmetric from the central $x_{1}-x_{3}$ plane), this shear is equal in absolute value but of contrary sign. The $\mathcal{T}_{x_{1} x_{3}}$ distributions are similar but they mainly occur on the corresponding perpendicular plane. Both shear stresses at the top vertical end have the same sign but are slightly smaller. In spite of their relative low value, the contribution of these two shears is significant since they are approximately twice as influential as the normal ones for the Tresca combined stress.

Although to some extend $\mathcal{T}_{x_{1}}$ is higher, at steady-state the normal components are similar and their positive and negative values are also similar (first row). As mentioned, this is due to the almost equal temperature increment from $T_{0}$ of the top and bottom faces (see Fig. 8 top). Also, $\mathcal{T}_{x_{1} x_{3}}$ is larger than the other shear stress since its distortion is larger, see the left Fig. 10 of each pair.

At pulse removal (second row) all components are maximum; the average stress of the first two normal components (middle of the scale) shifts towards compressive but the pure bending stress remains the same; for $\mathcal{T}_{x_{3}}$ both bending and compression augment in absolute value. At one of the corners the highest value of 
53.3 MPa is located. The shear stresses also raise at that time and the differences between positive and negative values become almost nil due to the deformation symmetry.

The distributions at maximum overcooling (third row) tend to those of steady-state (first row) although the values are smaller, that is, the stresses are relieved after $t_{m x}$ and before $t_{s s 2}$. This reduction is logical since during this period $T_{c}$ is closer to $T_{0}$ than during steadystate. The exception is for compressions of $\mathcal{T}_{x_{3}}$, since most of the TE temperature (70\%, see Fig. 8 top) is still above $T_{0}$.

The two shear stresses maintain the same distribution at the three instants since the distortion shape is always very similar.

As a corollary of the previous stress plots, Fig. 12 shows the maximum (at any TE point) Tresca stress distributions during the complete dynamic process. This plot is similar to the absolute value of the overcooling from Fig. 9 thick line, although there is no inflection point at $t_{m n}$ and the dominant factor is the maximum $T$ inside the TE. During overheating the stresses slowly relax, since as commented the temperature difference with $T_{0}$ is reduced. After $40 \mathrm{~s}$ the stress level returns to a value only slightly higher than the steady-state $\mathcal{T}_{t r}=$ $24 \mathrm{MPa}$; to exactly recover this number more than $80 \mathrm{~s}$ of simulation are necessary.

In the 3D stress sketches it is obvious that the maximums are at the far corners of the bottom face with the compressive $\mathcal{T}_{x_{3}}$ as predominant contribution. Even for a moderate pulse the maximum $\mathcal{T}_{t r}$ at $t_{m n}$ is more than twice that of the steady-state, and almost reaches the admissible value. Note that the zone of very low stress in steady-state occupies most of the central part of the TE, but for the other instants it is restricted to the central bottom end (except the edges).

Finally, Fig. 13 shows the locus of pulse gains $P$ and durations $\Delta t_{p}$ combinations so that the maximum TE Tresca stress does not surpass $\mathcal{T}_{a d}$. For the shortest $l_{3}$ $=5.8 \times 10^{-3} \mathrm{~m}$ (length used through most of the paper) and a threshold $P=1.704$ any duration can be applied. This threshold has been obtained from the quotient of $I_{o p}$ and the intensity corresponding to $60 \mathrm{MPa}$ (Fig. 7 bottom): after all, the steady-state can be regarded as a pulse driven by $I_{o p}$ and $\infty \Delta t_{p}$. For $P>2.3$ the decrease of admissible $\Delta t_{p}$ becomes small, and for $P>5$ $\Delta t_{p}$ must be very short, almost a spike.

The reason for this asymptotic decrease is the increase of Joule effect caused by the additional electric energy introduced in the PT, that is, the area of the pulse. But the relationships are not by any means linear, as shown by the curves and Eqs. (2). The proportional- ity does not hold for pulses with $P$ below the threshold, even if the pulse has infinite energy when $\Delta t_{p} \rightarrow \infty$; for large values a new equilibrium state with $T_{c s s}^{\prime}>T_{c s s}$ is reached, and the temperature distribution of Fig. 8 would never be high enough to induce stresses that surpass $\mathcal{T}_{\text {ad }}$.

The curve trends are similar for other $l_{3}$, but the threshold gain is smaller or higher for shorter or longer TEs: 1.65, 1.69, 1.7, 1.73 listed in the figure. Again the values are obtained from curves similar to that of Fig. 7 bottom. As expected, the shorter the TE the steeper the tendency to allowable $\infty$ pulse duration.

The lower position of the curves of shorter lengths is due to two causes. The first is related to the fact that the longer the TE the lower $I_{o p}$ from Section 4; therefore for a fixed $P, \bar{I}_{p}$ is lower and the duration can be longer for the same additional energy. The second can be understood from $T\left(x_{3}\right)$ of Fig. 8 top: for a fixed $\bar{T}_{h}$ and shorter TE, the distribution will have higher slope and higher value. Then the deformations of Fig. 10 will be more pronounced and the swelling of the center will interact with the rotation of the TE side close to the cold face, increasing the maximum stresses. Both causes are then inversely proportional to $l_{3}$, see Eq. (6) for an approximation of the first.

\section{Conclusions}

In the present article, a preexisting multi-coupled, three-dimensional and non-linear research finite element code has been extended and applied to the study the elasto-thermoelectric interactions in pulsed thermoelectric devices. The coupling between mechanical and thermal fields is two-way, although the influence of the mechanical towards thermal response is small. The important aspect of the optimal intensity to be prescribed for maximum overcooling is solved with analytical iterations and finite element bisections.

Special care has been taken to the not much studied aspect of strain and stress distributions in the thermoelement and in the consideration of the dependency of fundamental material properties with the temperature. The code is validated with a simple and specially developed analytical expression, and with experimental results taken from the literature.

Several conclusions can be stated, some already published (although sometimes not completely justified) and others new:

- Due to symmetries and repetitions only one a half of a thermocouple is to be be analyzed. But the 


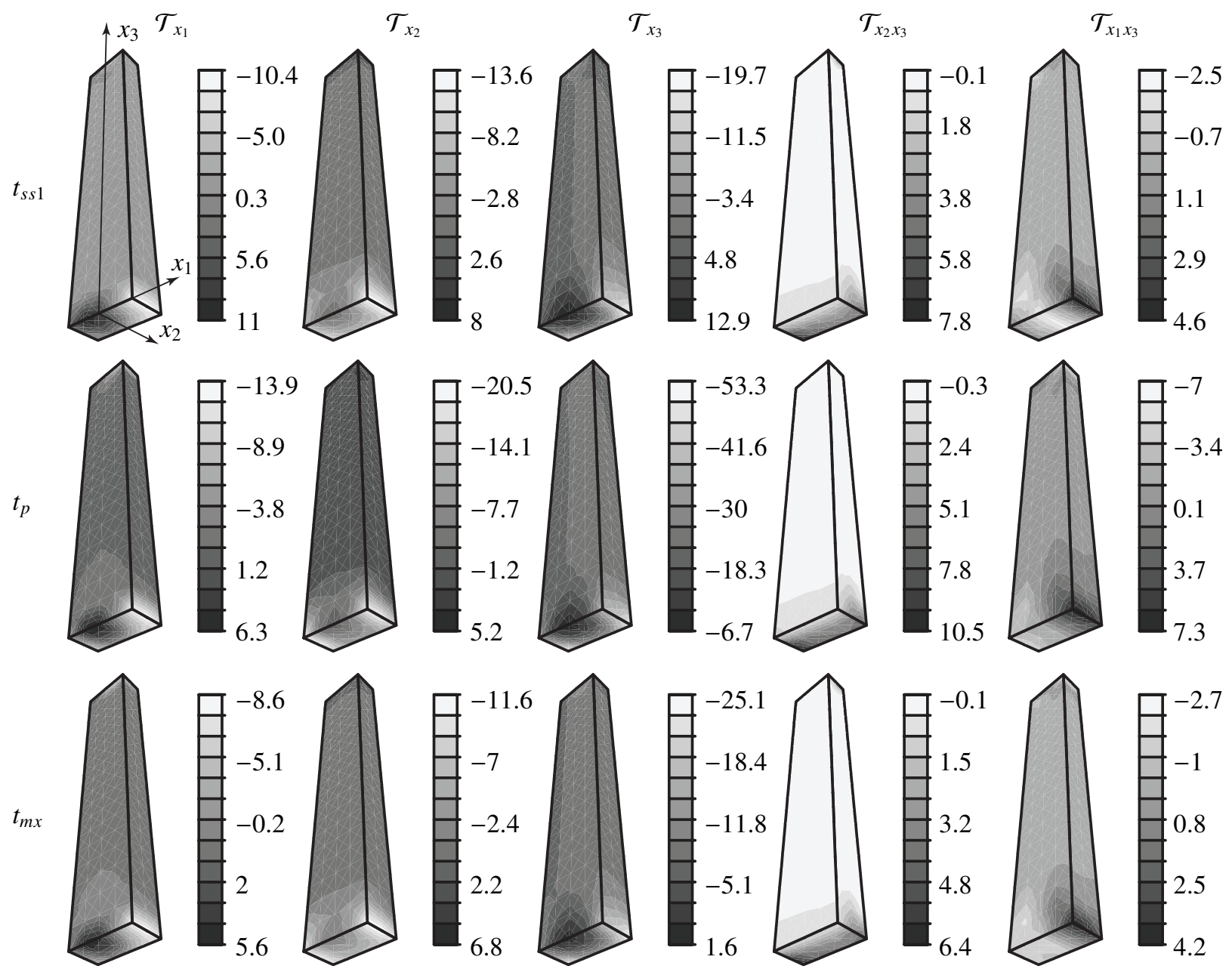

Figure 11: Plots for five stress components (columns) in MPa inside a thermoelement at steady-state (top), pulse removal (middle) and maximum overheating (bottom). Finite elements with variable properties, visible vertical plane $x_{1}-x_{3}$ symmetric and view from the cold face.

three-dimensionality of the cell must be preserved, at least for the study of stresses.

- The optimal intensity given from simple analytical formulae is not accurate enough, unless a good average temperature can be guessed.

- High pulse gains should not be applied, since there is a practical limit in the overcooling that can be reached and in temperature and stress limits.

- The dependency of properties on temperature must be included since the Thomson effect significantly increases cooling and reduces overheating.

- The non-thermoelectric parts (alumina, copper, tin solder) are fundamental for thermal dynamics and stress calculations. The smaller the mass of these materials the better the performance will be.
- The electric pulse causes high thermal stresses due to overheating, a detrimental situation since thermoelectric materials have low mechanical strengths specially under tension.

- The stress distributions are complex and they tend to concentrate in the thermoelement external corners close to the cold and hot faces for all instants.

- The predominant stress component is the vertical one for the studied boundary conditions, although the others, particularly the shear stresses, play a certain role in failure.

- Maximum stresses occur at the end of the pulse; the subsequent overheating in fact releases stresses from the steady-state situation.

- In normal functioning, under pulses the stresses are high in the thermoelement. Repeated application 


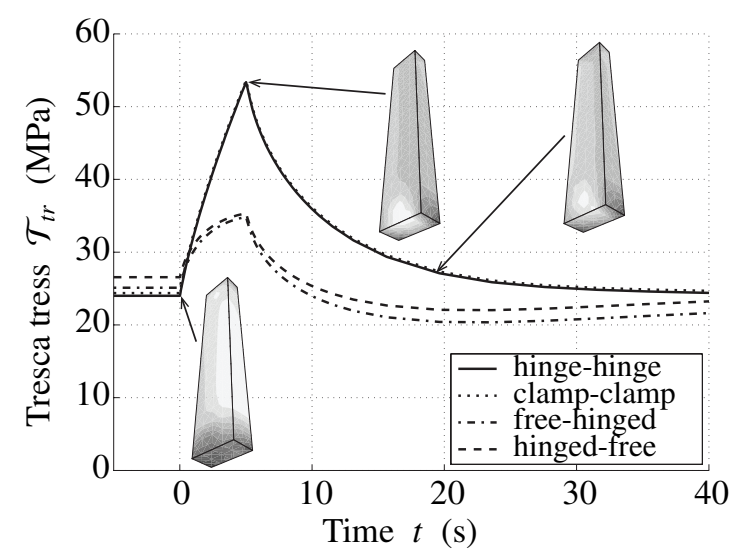

Figure 12: Maximum Tresca stress (white for zero stress, black for maximum) for thermoelectric transient process with finite element variable properties. Tresca stress plot: figures from left to right at steady-state, pulse removal, maximum overheating.

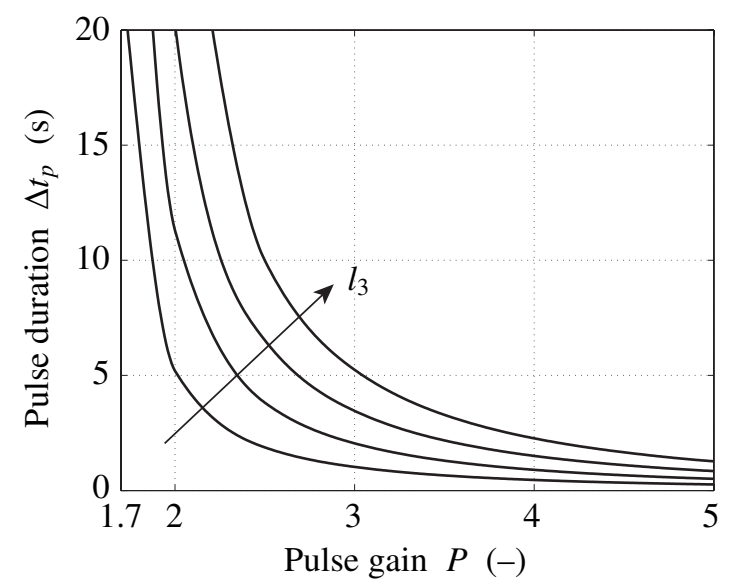

Figure 13: Locus of combinations pulse gain versus duration that do not surpass admissible stress (see Table 1). Lengths of thermoelement $3.4,4.6,5.8,7.0 \times 10^{-3} \mathrm{~m}$.

of pulses would probably create fatigue failure.

- The non-thermoelectric materials are subjected to even higher stresses, above the admissible. An accurate analysis will require consideration of plasticity and relaxation, object of an ongoing work.

- Combinations of pulse gain and duration should not be surpassed to mechanically protect the thermoelement; a $P$ threshold variable with the TE length exists so that any time can be applied.

The current results will permit to further study PTs and to optimally design them controlling their functional requirements, for example, maximizing the holding time, minimizing the post-pulse temperature, etc. The calculations could be incorporated in the future into a software package able to choose the pulse shape and the thermoelement geometry taking into consideration the application.

\section{Acknowledgments}

This research was partially supported by grants CSD2008-00037 Canfranc Underground Physics and Polytechnic University of Valencia under programs PAID 02-11-1828 and 05-10-2674.

\section{References}

[1] S.B. Riffat and X. Ma. Thermoelectrics: a review of present and potential applications. Applied Thermal Engineering, 23:913935, 2003.

[2] L.S. Stilbans and N.A. Fedorovich. The operation of refrigerating thermoelectric elements in nonstationary conditions. Sov. Phys. Tech. Phys., 3:460, 1958.

[3] R.J. Buist and P.G. Lau. Theoretical analysis of thermoelectric cooling performance enhancement via thermal and electric pulsing. In Fifteenth International Conference on Thermoelectrics, 1996.

[4] G.J. Snyder, J.P. Fleurial, and T. Caillat. Supercooling of Peltier cooler using a current pulse. Journal of Applied Physics, 92(3):1564-1569, 2002.

[5] K. Landecker and A.W. Findlay. Study of the fast transient behaviour of Peltier junction. Solid-State Electronics, 3:239-260, 1961.

[6] A. Chakraborty and K.C. Ng. Thermodynamic formulation of temperature-entropy diagram for the transient operation of a pulsed thermoelectric cooler. International Journal of Heat and Mass Transfer, 49:1845-1850, 2006.

[7] T. Thonhauser and G.D. Mahan. Improved supercooling in transient thermoelectrics. Applied Physics Letters, 85(15):32473249, 2004.

[8] R. Yang, G. Chen, A.R. Kumar, G.J. Snyder, and J.P. Fleurial. Transient cooling of thermoelectric coolers and its applications for microdevices. Energy Conversion and Management, 46:1407-1421, 2005.

[9] D. Astrain, J.G. Vián, and M. Domínguez. Increase of COP in the thermoelectric refrigeration by the optimization of heat dissipation. Applied Thermal Engineering, 23:2183-2200, 2003.

[10] Y.H. Cheng and W.K. Lin. Geometric optimization of thermoelectric coolers in a confined volume using genetic algorithms. Applied Thermal Engineering, 25:2983-2997, 2005.

[11] Y.H. Cheng and C. Shih. Maximizing the cooling capacity and COP of two-stage thermoelectric coolers through genetic algorithm. Applied Thermal Engineering, 26:937-947, 2006.

[12] M. Jaegle, M. Bartel, D. Ebling, A. Jacquot, and H. Bottner. Multiphysics simulation of thermoelectric systems. In European Conference on Thermoelectrics ECT2008, 2008.

[13] M. Jaegle. Multiphysics simulation of thermoelectric systems modeling of Peltier - cooling and thermoelectric generation. In Proceedings of the COMSOL, 2008.

[14] S. Turenne S, T. Clin, D. Vasilevskiy, and R.A. Masut. Finite element thermomechanical modeling of large area thermoelectric generators based on bismuth telluride alloys. J. Electron Mater, 39:1926-1933, 2010.

[15] M. Picard, S. Turenne, D. Vasilevskiy, and R.A. Masut. Numerical simulation of performance and thermomechanical behavior 
of thermoelectric modules with segmented bismuth-telluridebased legs. J. Electron Mater, 42:2343-2349, 2013.

[16] GY. Wu, T. Ming, X. Li, T. Pana, K. Peng, and X. Luo. Numerical simulations on the temperature gradient and thermal stress of a thermoelectric power generator. Energy Conversion and Management, 88:915-927, 2014.

[17] J.L. Pérez-Aparicio, R.L. Taylor, and D. Gavela. Finite element analysis of nonlinear fully coupled thermoelectric materials. Computational Mechanics, 40:35-45, 2007.

[18] R. Palma, J.L. Pérez-Aparicio, and R.L. Taylor. Non-linear finite element formulation applied to thermoelectric materials under hyperbolic heat conduction model. Computer Method in Applied Mechanics and Engineering, 213:93-103, 2012.

[19] R. Palma, J.L. Pérez-Aparicio, and R. Bravo. Study of hysteretic photovoltaic behavior using the finite element method, extended thermodynamic and inverse models. Energy Conversion and Management, 65:557-563, 2013.

[20] J.L. Pérez-Aparicio, R. Palma, and R.L. Taylor. Finite element analysis and material sensitivity of Peltier thermoelectric cells coolers. International Journal of Heat and Mass Transfer, 55:1363-1374, 2012.

[21] J.L. Pérez-Aparicio, R. Palma, and R.L. Taylor. Multiphysics and thermodynamic formulations for equilibrium and nonequilibrium interactions: non-linear finite elements applied to multi-coupled active materials. Archives of Computational Methods in Engineering, (DOI 10.1007/s11831-015-9149-9):149, 2016.

[22] R. Palma, J.L. Pérez-Aparicio, and E. Moliner. Elastothermoelectric beam formulation for modeling thermoelectric devices. Energy Conversion and Management, In preparation, 2016.

[23] J.L. Pérez-Aparicio, P. Moreno-Navarro, and J.J. GómezHernández. Optimization of pulsed thermoelectrics' geometry with simulated annealing and non-linear finite elements. Applied Thermal Engineering, In preparation, 2016.

[24] P. Moreno-Navarro, J.L. Pérez-Aparicio, and J.J. GómezHernández. Optimization of thermoelectric pulses driven by intensity or voltage, using simulated annealing and non-linear finite elements. Applied Thermal Engineering, In preparation, 2016.

[25] R.L. Taylor. FEAP A Finite Element Analysis Program: User Manual. University of California, Berkeley, 2010. http://www.ce.berkeley.edu/feap.

[26] Laird Technologies. THR-BRO-Thermoelectric-Hndbk 0110 Laird Technologies, 2010. http://www.lairdtech.com.

[27] D.M. Rowe, editor. CRC Handbook of Thermoelectrics. CRC Press, 1995.

[28] Th. Clin, S. Turenne, D. Vasilevskiy, and R.A. Masut. Numerical simulation of the thermomechanical behavior of extruded bismuth telluride alloy module. Journal of Electronic Materials, 38(7):994-1001, 2009.

[29] IEEE Standards Board. IEEE Standard on Piezoelectricity, 1987.

[30] O.C. Zienkiewicz, R.L. Taylor, and J.Z. Zhu. The Finite Element Method: The Basis, 7th ed. Elsevier Butterworth-Heinemann, 2013.

[31] J. Abate and P.P. Valkó. Multi-precision Laplace transform inversion. International Journal for Numerical Methods in Engineering, 60(5):979-993, 2004.

[32] W. Seiffert, M. Ueltzen, and E. Muller. One-dimensional modelling of thermoelectric cooling. Physica Status Solidi A, 194(1):277-290, 2002.

[33] Q. Zhou, Z. Bian, and A. Shakouri. Pulsed cooling of inhomogeneous thermoelectric materials. Journal of Physics D: Applied Physics, 40:4376-4381, 2007.
[34] J.L. Gao, Q.G. Du, X.D. Zhang, and X.Q. Jiang. Thermal stress analysis and structure parameter selection for a Bi2Te3based thermoelectric module. Journal of Electronic Materials, 40(5):884-888, 2011. 